\title{
Move over Blood Pressure: Make Room for Cardiorespiratory Fitness as a Vital Sign
}

\author{
Nina Radford
}

\begin{abstract}
Cardiorespiratory fitness (CRF) describes the ability of an individual to perform physical exercise, an activity that relies on the cardiovascular system's capacity to facilitate oxygen delivery to working muscles and the pulmonary system's ability to clear carbon dioxide from the blood. Ideally, CRF is measured using cardiopulmonary stress testing (exercise with analysis of gas exchange). To enhance the feasibility of assessing CRF in large populations, prediction equations using only exercise variables were developed to provide measures of estimated CRF. More recently, nonexercise methods of assessing CRF have been developed. Using exercise (objective) methods of measuring CRF, a robust evidence base has developed describing the inverse association of CRF with total mortality, nonfatal cardiovascular events, incident cancer, cancer survival, chronic conditions, and dementia; CRF has also been shown to be inversely related to development of cardiac risk factors including hypertension. Because CRF provides a strong, graded inverse association with cardiovascular and all-cause mortality, recent American Heart Association scientific statements have vigorously promoted the concept of CRF as a vital sign: All adults should have an estimation of CRF included in their annual health care visits along with resting blood pressure (BP), heart rate, and body mass index (BMI). The addition of CRF as a vital sign provides enhanced risk prediction that can improve patient care and encourage the incorporation of physical activity (PA) programs into treatments plans aimed at improved health outcomes.
\end{abstract}

Keywords: Cardiorespiratory fitness, Cardiovascular disease, Hypertension, Physical activity, Prevention.

How to cite this article: Radford N. Move over Blood Pressure: Make Room for Cardiorespiratory Fitness as a Vital Sign. Hypertens J 2017;3(2):51-57.

Source of support: Nil

Conflict of interest: None

\section{INTRODUCTION}

Over the last 30 years, a vast literature has developed describing the inverse association of CRF and numerous health outcomes including total mortality, nonfatal cardiovascular events, incident cancer, cancer survival, chronic

Clinical Cardiologist

Cooper Clinic, Dallas, Texas, USA

Corresponding Author: Nina Radford, Clinical Cardiologist Cooper Clinic, Dallas, Texas, USA, Phone: +9725602741, email: nbradford@cooper-clinic.com conditions, and dementia; CRF has also been shown to be inversely related to development of individual and clustered cardiovascular risk factors; these associations have been demonstrated in the young and old, healthy and unhealthy, men and women, and in different racial/ ethnic groups. ${ }^{1-16}$ Because CRF provides a strong, graded inverse association with cardiovascular and all-cause mortality, recent American Heart Association scientific statements have vigorously promoted the concept of CRF as a vital sign. ${ }^{17-19}$ In clinical practice, all adults would have an estimation of CRF included in their annual health care examinations much like measurements of resting $\mathrm{BP}$, heart rate, and BMI. To enhance the feasibility of this recommendation, both exercise- and nonexercise-based methods are available to estimate CRF. In this context, the goals of this paper are: (1) To define CRF and its relationship to PA, (2) to review the role of CRF in preventing hypertension and modifying cardiovascular risk in the setting of established hypertension, and (3) to describe CRF assessment and utilization in clinical practice.

\section{DEFINITION AND DETERMINANTS OF CRF}

The CRF describes the ability of an individual to perform physical exercise, an activity that relies on the cardiovascular system's capacity to facilitate increased oxygen uptake needed by working muscles and the pulmonary system's ability to clear carbon dioxide from the blood (diffusion) and disperse into the atmosphere (ventilation). The gold standard for measuring CRF is cardiopulmonary exercise testing because it provides the most accurate and standardized quantification of CRF. ${ }^{20}$ Typically, exercise is performed on a motorized treadmill; a stationary cycle ergometer can also be used if the individual has an unsteady gate or orthopedic limitations to walking, but leg fatigue may drive termination of exercise at a submaximal effort in deconditioned individuals. ${ }^{21}$ A mouthpiece and nose clip or facemask are worn throughout exercise and are connected to a gas analyzer. Cardiopulmonary exercise testing combines conventional exercise stress-testing variables (electrocardiography changes, heart rate and BP responses, heart rhythm abnormalities, and exercise-related symptoms) with ventilatory expired gas analysis (providing measures of oxygen uptake $\left(\mathrm{VO}_{2}\right)$, carbon dioxide output, and minute ventilation). 
Cardiopulmonary exercise testing generates a number of variables that have characteristic patterns in the setting of health and in specific disease states. ${ }^{19}$ A key measure provided by this test is maximal oxygen uptake $\left(\mathrm{VO}_{2 \max }\right.$ or $\mathrm{VO}_{2 \text { peak }}$ ); this value defines maximal aerobic capacity in generally healthy individuals or peak exercise capacity in individuals with cardiac or pulmonary disease. The $\mathrm{VO}_{2 \max }$ is usually expressed in milliliters of $\mathrm{O}_{2}$ per kilogram of body weight per minute (to allow comparisons among individuals with different body weights); values can range from $80 \mathrm{~mL} \mathrm{O}_{2} / \mathrm{kg} /$ minute in a young, elite athlete to 40 $\mathrm{mL} \mathrm{O}_{2} / \mathrm{kg} /$ minute in a sedentary 35-year-old to $<10 \mathrm{~mL}$ $\mathrm{O}_{2} / \mathrm{kg} /$ minute in a patient with end-stage lung disease. ${ }^{20,21}$

The $\mathrm{VO}_{2 \max }$ (measured CRF) is influenced by age, sex, genetics, PA, lifestyle choices (diet, smoking), physiological factors (fat, muscle mass), exercise training, and the presence of clinical or subclinical (cardiac, pulmonary, and skeletal muscle) disease. ${ }^{18,20-23}$ In a study of 473 sedentary adults, from 99 families enrolled in the Health, Risk Factors, Exercise Training, and Genetics (HERITAGE) Family Study, participants exposed to a standardized 20-week exercise program gained increases in $\mathrm{VO}_{2 \max }$ of 15 to $25 \%$; using a panel of 21 single nucleotide polymorphisms, investigators were able to estimate that almost half of the gain in CRF could be attributed to favorable genetic inheritance. $^{22}$

Cardiopulmonary exercise testing with its requisite ventilatory expired gas analysis require specialized equipment and higher levels of staff training; it may be a burdensome procedure for some individuals undergoing testing and cardiopulmonary exercise testing facilities may not be broadly available. Historically, from an epidemiological point of view, obtaining measured CRF data from large populations was difficult, if not impossible. To solve this problem, prediction equations were developed so that estimated CRF could be derived from treadmill variables alone. Exercise-based estimates of CRF are obtained using common treadmill protocols (Bruce, Balke, Modified Balke) or cycle ergometer exercise without the complexity of measuring expired gases. The CRF is estimated from the work rate achieved during exercise and is commonly expressed in units of metabolic equivalents (METs). One MET is defined as the energy expenditure required for sitting quietly (approximately $3.5 \mathrm{~mL} \mathrm{O}_{2} / \mathrm{kg} /$ minute $\mathrm{mL}$ for an average adult). To put this in a clinical context, moderate-intensity activities are those that result in an energy expenditure three to six times the energy rquired to sit quietly or 3 to 6 METS.

These prediction equations are less accurate if the patient is allowed to hold onto the treadmill handrails (because this decreases workload and increases exercise duration) or if there is patient-protocol mismatch. The patient's fitness should be matched with treadmill protocol (e.g., choosing a slower belt speed and rate of incline for an unfit patient). These caveats are important to keep in mind as we consider adding measured CRF as a vital sign. $17,18,20$

Estimated CRF derived from the peak work rate is the typical measure of CRF in most epidemiological studies involving large populations. Almost 30 years ago, Blair et $\mathrm{al}^{7}$ published a landmark study assessing estimated CRF obtained using maximal treadmill exercise testing and risk of all-cause and cause-specific mortality in 10,224 men and 3,120 women enrolled in the Aerobics Center Longitudinal Study (ACLS). With an average follow-up of more than 8 years, adjusted all-cause mortality rates declined across CRF quintiles from the least fit to the most fit. Lower mortality rates in higher fitness categories also were seen for cardiovascular disease and cancer.

\section{CARDIORESPIRATORY FITNESS VS PHYSICAL ACTIVITY}

Because CRF can be modified through changes in routine PA, it is regarded as a surrogate for habitual PA. Why not add PA as a vital sign rather than CRF? This seems like a much easier variable to collect. Furthermore, clinical recommendations aimed at improving health are made in terms of PA with the goal of improving CRF.

First, as a clinical variable, PA can be difficult to quantify in an accurate and reproducible way. ${ }^{24,25}$ Historically, much of the epidemiological data evaluating the association of PA with health outcomes have quantified PA using self-report questionnaires. These assessments can be subjective regarding the duration, intensity, and frequency of PA. To obtain more objective measures of PA, investigators are now using accelerometers to record PA throughout the day. However, little research has been published on how PA assessed by accelerometers will perform in predicting health outcomes compared with data collected by PA questionnaire.

Most importantly, CRF has many determinants of which PA is just one. An individual may engage in regular PA and still have low CRF (unfit) because of poor lifestyle choices (smoking) or the presence of subclinical or clinical disease (emphysema); quitting smoking may be the best route to improved CRF in this individual.

A number of studies have evaluated the relationship between PA, CRF, and mortality. Lee et $\mathrm{al}^{26}$ examined the combined associations and relative contributions of PA and CRF to all-cause mortality in 31,818 male and 10,555 female participants in the ACLS with PA assessed by selfreported questionnaire and CRF assessed by maximal treadmill test. In this generally healthy population, CRF was more strongly associated with all-cause mortality than PA. Myers et $\mathrm{al}^{27}$ reported all-cause mortality with 
5 years of follow-up in a subgroup of 842 less healthy male veterans from Palo Alto, California with estimated CRF from clinically indicated treadmill tests (for chest pain, dyspnea, and so on) and habitual PA obtained by self-report questionnaire. In this high risk group of men, CRF was also a stronger predictor of mortality than PA. Davidson et $\mathrm{al}^{28}$ evaluated a larger cohort $(8,171)$ of male veterans from Washington, DC with similar methods estimating CRF and PA, who were followed for 8.7 years, for all-cause mortality. The PA was a significant predictor of mortality after controlling for clinical variables; however, the association was eliminated after further adjusting for CRF. Conversely, CRF remained a strong predictor of mortality, independent of PA status and established CV risk factors. These findings support the concept that CRF mediates the association between PA and mortality. Thus, the recommendation that $\mathrm{CRF}$, a better marker of risk than PA, ought to be included as a vital sign.

Given that the process of atherosclerosis begins in youth, continues for decades, culminating in clinical cardiovascular events, it is reasonable to suggest that both CRF and PA play important roles in primordial prevention (CRF preventing the development of $\mathrm{CV}$ risk factors) and primary prevention (PA interventions designed to modify low CRF with the goal of preventing an initial CVD event). This concept is well illustrated in the case of hypertension.

\section{CARDIORESPIRATORY FITNESS AND HYPERTENSION}

The presence of an age-related increase in BP is well documented. Data from 2,000 enrollees in the Framingham Heart Study demonstrated that in normotensive and untreated hypertensive subjects, systolic BP increased linearly from age 30 through age 84 with a current rise in diastolic BP until age 50 to 60 after which time it. ${ }^{29}$ Furthermore, the age-related progressive increase in BP is accompanied by an incremental increase in cardiovascular risk evident beyond BP levels of $115 / 75 \mathrm{~mm} \mathrm{Hg}{ }^{30}$ Whether the age-related increase in BP is inevitable is a matter of debate. It is likely the result of a combination of innate biological aging, genetic underpinning, and lifestyle choices. ${ }^{31,32}$ Some of the age-related increases in hypertension can be attenuated through lifestyle modifications that focus on dietary salt restriction, high consumption of fruits and vegetables, increased intake of fish, reduced intake of saturated fat, maintaining an ideal body weight, engaging in regular PA, and not smoking. 33,34

A number of large prospective cohort studies have examined the cross-sectional and/or longitudinal relationships between CRF and the risk of developing hypertension in a variety of populations: generally healthy white men and women, men and women with a family history of hypertension, young black and white men and women with long term follow-up, and older black and white men and women at high risk for CVD events. Barlow et al ${ }^{35}$ evaluated almost 5,000 normotensive, generally healthy women followed in the ACLS; the cumulative incidence of hypertension was $3.2 \%$ with an average follow-up of 5 years. High-fit women had one-third the risk of developing hypertension compared with low-fit women. Liu et $\mathrm{al}^{36}$ studied 13,953 generally healthy normotensive men followed in the ACLS. High-fit men had lower systolic BP during the follow-up period compared with moderateand low-fit men. Shook et $\mathrm{al}^{37}$ evaluated the joint association of CRF and family history of hypertension on incident hypertension in more than 6,000 normotensive men and women in the ACLS. Thirty-three percent reported a parent with hypertension. Low-fit individuals with parental hypertension had a $70 \%$ higher risk for developing hypertension and high-fit individuals with parental history had only a $16 \%$ increased risk of developing hypertension (using high-fit individuals with no parental hypertension as the referent group).

Carnethon et $\mathrm{al}^{38}$ evaluated 4,681 black and white men and women enrolled in The Coronary Artery Risk Development in Young Adults (CARDIA) Study (average age 25), who were followed for over 20 years. Across race-sex groups, there was an inverse, graded association between CRF and the development of hypertension. The estimated proportion of hypertension cases that could be prevented if participants moved to a higher fitness category (i.e., preventive fraction) was $34 \%$. Juraschek et a ${ }^{39}$ examined the association of fitness with prevalent and incident hypertension in 57,284 less healthy black and white men and women from The Henry Ford Exercise Testing (FIT) Project (1991-2009). Fitness was measured using a clinician-referred treadmill stress test (with indications such as chest pain, "rule out" ischemia, and shortness of breath). In this group at high risk for CVD events (more than $40 \%$ current smokers and about half with a family history of heart disease), higher CRF was significantly associated with lower rates of hypertension at baseline.

Prehypertension, affecting 25 to $50 \%$ of adults worldwide, increases the risk of incident hypertension by two- to three-fold and is an independent risk factor for cardiovascular disease. The optimal treatment strategies for prehypertension are controversial. ${ }^{40}$ Drug therapy has been shown to reduce cardiovascular events among individuals with prehypertension who have clinical cardiovascular disease with or without diabetes; this risk reduction needs to be weighed against drug-related adverse events including hyperkalemia, renal failure, and hypotension as well as the cost associated with drug 
treatment in this patient population. ${ }^{41,42}$ Treatment strategies are uncertain in generally healthy individuals with prehypertension.

Clinical variables associated with progression from prehypertension to hypertension include black ethnicity, older age, higher BMI, diabetes, chronic kidney disease, and low CRF. ${ }^{40}$ In the case of CRF, the impact of high fitness on progression is substantial. Faselis et $\mathrm{al}^{43}$ evaluated 2,303 male veterans with prehypertension followed for 9.2 years; CRF was a strong and independent predictor of the rate of progression. Compared with the high-fit individuals (10.0 METs), the adjusted risk for developing HTN was $72 \%$ higher for the least-fit individuals. Other significant predictors of progression were age, resting systolic BP and type II diabetes mellitus.

Finally, the impact of change in CRF (e.g., low-fit to high-fit over time) on the risk of developing hypertension has been evaluated in the few longitudinal studies with repeated measures of CRF. Three studies are presented here with approximately 5, 10, and 20-year follow-up. Sui et $\mathrm{al}^{44}$ reported on 4,932 men and women in the ACLS who had at least four preventive medicine evaluations and were free of hypertension during the first three examinations with almost 11 years of follow-up. Among 4,932 participants, 1,954 developed hypertension. There were five patterns of changing CRF over time: Decreasing, increasing, bell-shaped, U-shaped, and inconsistent. A pattern of increasing CRF provided the lowest risk of hypertension. Jae et $\mathrm{al}^{45}$ evaluated 3,831 men without CVD, hypertension, or diabetes, who were seen at the Samsung Medical Center in Seoul, South Korea, for two health examinations. The CRF was directly measured by peak oxygen uptake using expired gas analysis during a standard treadmill test. During an average follow-up of 5 years, $373(9.7 \%)$ subjects developed hypertension. The incidence of hypertension was inversely associated with baseline CRF quartiles. In a study with much longer follow-up time, Jae et $\mathrm{al}^{46}$ evaluated Finnish 431 men without hypertension enrolled in the Kuopio Ischemic Heart Disease Study with measures of CRF at baseline and 11 years later with follow-up for hypertension 10 years after that. Men who demonstrated the largest decline in CRF had more than a four-fold risk of incident hypertension compared with men with the smallest decrease or improvement in CRF. Collectively, these studies underscore the potential importance of CRF to prevent the aging-related progressive rise in $\mathrm{BP}$ as well as the development of prehypertension and hypertension.

In addition to its inverse association with incident hypertension, several large epidemiological studies have demonstrated an inverse and independent association of CRF and mortality risk in individuals with established hypertension. Myers et $\mathrm{al}^{47}$ studied a total of 6,213 male veterans in Palo Alto, California, who referred for treadmill exercise testing for clinical reasons and were followed for 6 years; 3,679 had an abnormal exercisetest result or a history of cardiovascular disease, or both, and 2,534 had a normal exercise-test result and no history of cardiovascular disease. They found that CRF was a more powerful predictor of mortality than other established risk factors for cardiovascular disease. In all subgroups defined according to risk factors (including the subgroup of hypertensives), the risk of death from any cause in low-fit men ( $<5 \mathrm{METs})$ was roughly double that of moderate-fit men $>8$ METs. Faselis et al ${ }^{48}$ studied a group of 4,183 hypertensive veterans from Washington DC referred for treadmill exercise testing for clinical indications to evaluate the interaction between fitness, fatness, and mortality risk in hypertensive individuals. They found that increased CRF was associated with lower mortality risk in hypertensive males regardless of BMI. Finally, in a study that included men and women, Evenson et $\mathrm{al}^{49}$ evaluated the effect of CRF on mortality among hypertensive and normotensive women $(2,712)$ and men $(3,000)$ followed up for more than 20 years in the Lipids Research Clinics Prevalence Study. Comparing the lowest with the highest quintile of fitness, the adjusted all-cause mortality among hypertensive women was 1.7 and among hypertensive men was 2.0.

How improved CRF reduces the risk of all-cause mortality in a number of clinical settings, including hypertension, is not fully understood. From a clinical perspective, higher levels of CRF are associated with reduced levels of traditional cardiovascular risk factors, such as hypertension, type II diabetes, metabolic syndrome, lipid profiles, fasting blood glucose, and anthropometric measures. Each of these effects is mediated through multiple downstream modifications; e.g., increasing CRF and decreasing BP are mechanistically linked to alterations in vascular mechanics. Improvements in metabolic factors with increasing CRF are likely related to CRF-mediated increases in insulin sensitivity and reduced levels of inflammation and oxidative stress. Improvements in cardiovascular risk may also be mediated by changes in vascular health mediated through nitric oxide function and or changes in autonomic nervous system activity. Because CRF relies on the integrated function of multiple physiological systems, it reflects the global health status of an individual, and as such serves as an important vital sign. ${ }^{50-54}$

\section{CARDIORESPIRATORY FITNESS IN CLINICAL PRACTICE}

In clinical practice, an estimation of CFR could provide an additional tool for risk stratification when making 
clinical decisions. In situations where there is an intermediate determination of risk, the presence of low CRF may lend support for a decision to initiate drug treatment of hypertension or dyslipidemia for example. An annual estimation of CRF could also be a means to assess the impact of a PA program. Experts suggest that measurement of CRF in clinical settings is feasible and no more difficult than measuring $\mathrm{BP}^{18}{ }^{18}$ They recommend that at a minimum, all adults should have CRF estimated each year using a nonexercise algorithm (although they caution that this should not be viewed as replacement for an objective measure of CRF). Ideally, all adults (age range yet to be determined) should have CRF estimated using a maximal exercise test on a regular basis (follow-up interval yet to be determined) similar to other preventative services like mammography or colonoscopy. Finally, adults with chronic disease should have CRF measured with a peak or symptom-limited cardiopulmonary stress test on a regular basis.

Numerous nonexercise equations to estimate CRF have been developed over the last 10 to 15 years using cross-sectional data from generally healthy cohorts over a range of ages and cohort sizes (as few as 100 and as many as 46,000 ) using variables of age, sex, body weight (or BMI, percentage of body fat, waist circumference), PA/exercise/ training (self-reported or measured), smoking, resting $\mathrm{HR}$ and/or perceived functional ability. ${ }^{18}$ In a recent study designed to determine the validity of nonexerciseestimated CRF on mortality, Nauman et $\mathrm{al}^{55}$ assessed the predictive value of estimated CRF (eCRF) in healthy men $(\mathrm{N}=18,721)$ and women $(\mathrm{N}=19,759)$ aged 30 to 74 years, who were enrolled in a large health survey in Norway (Nord-Trondelag Health Study [HUNT]), with median follow-up of 16 years. They found that low eCRF was independently associated with CVD and all-cause mortality. The inclusion of traditional clinical CVD risk factors added little to risk discrimination and did not improve the classification of risk beyond this simple eCRF measurement.

Their nonexercise algorithm-estimated eCRF used the following algorithms:

$$
\begin{aligned}
& \text { Women: } 78.00-(0.297 \times \text { Age })-(0.270 \times W C)-(0.110-r H R) \\
& +(2.674 \times \mathrm{PA})
\end{aligned}
$$

The WC is waist circumference. The rHR is resting heart rate. The PA variable is assigned a value of 1 , if they were meeting recommended levels of PA: Vigorousintensity exercise training for $\geq 20$ minutes per day on $\geq 3$ days per week for a total of $\geq 75$ minutes per week and/ or moderate-intensity exercise training for $\geq 30$ minutes per day on $\geq 5$ days per week for a total of $\geq 150$ minutes per week. The PA is assigned a value of 0 , if they are not meeting recommended levels of PA.
The overarching goal of measuring or estimating CRF is to identify the lowest CRF or least-fit individual because the greatest reduction in total mortality occurs between the least-fit ( $<5 \mathrm{METs})$ and the next least-fit levels of CRF (5 to 7 METs). Getting out of the lowest category of fitness has a much greater impact on health outcomes than moving from good (10-12 METs) to better (>13 METs) fitness categories. high-fit individuals (those with CRF > 13 METS), increases in CRF have less impact on improving mortality and more impact on improving performance.

A PA plan for a low-fit individual needs to deliver a sufficient dose of exercise to increase CRF 1 or 2 METs; this is associated with $10 \%$ to $30 \%$ lower cardiovascular risk. When performed frequently over weeks or months, a wide variety of PA programs produce this level of CRF improvement in most adults. The PA programs need to include exercise activities that involve major muscle groups working in a continuous and rhythmic nature, such as brisk walking, jogging, running, cycling, swimming, and so on. Standing at the net for most of a doubles tennis game is not sufficient exercise intensity to advance fitness. Light-to-moderate intensity activity is recommended for deconditioned or the elderly while moderate and/or vigorous intensity activity is recommended for most generally healthy adults. Advancing exercise intensity, rather than frequency or duration, is more likely to increase CRF. Individuals ought to accumulate 150 minutes per week of light-to-moderate or moderate-intensity exercise and 75 minutes per week of vigorous-intensity exercise (or some combination of both; exercise sessions can be as short as 10 minutes. ${ }^{56}$

In conclusion, there are many compelling indications to measure CRF as a vital sign. That being said, the integration of estimates of CRF into routine clinical practice may be challenging. Referring this testing to cardiovascular subspecialists, especially in generally healthy individuals, may not be an appropriate utilization of subspecialty expertise. Understandably, primary care providers outside of academic centers may be resistant to perform treadmill testing to estimate CRF citing lack of time, training, equipment, and reimbursements. Specialty teams of exercise physiologists or other health care providers may be needed to fill this function. In the meantime, familiarizing primary care providers with nonexercise algorithms to estimate CRF (on a smartphone app or embedded in an electronic medical record) may be an important first step in adding CRF as a vital sign.

\section{REFERENCES}

1. Harber MP, Kaminsky LA, Arena R, Blair SN, Franklin BA, Myers J, Ross R. Impact of cardiorespiratory fitness on allcause and disease-specific mortality: advances since 2009. Prog Cardiovasc Dis 2017 Jun-Jul;60(1):11-20. 
2. Al-MallahMH, QureshiWT, KeteyianSJ, BrawnerCA, Alam M, Dardari Z, Nasir K, Blaha MJ. Racial differences in the prognostic value of cardiorespiratory fitness (results from the Henry Ford Exercise Testing Project). Am J Cardiol 2016 May 1;117(9):1449-1454.

3. Kokkinos P, Myers J, Faselis C, Panagiotakos DB, Doumas M, Pittaras A, Manolis A, Kokkinos JP, Karasik P, Greenberg M, et al. Exercise capacity and mortality in older men: a 20-year follow-up study. Circulation 2010 Aug 24;122(8):790-797.

4. McAuley P, Myers J, Emerson B, Oliveira RB, Blue CL, Pittsley J, Froelicher VF. Cardiorespiratory fitness and mortality in diabetic men with and without cardiovascular disease. Diabetes Res Clin Pract 2009 Sep;85(3):e30-e33.

5. DefinaLF, Willis BL, Radford NB, Gao A, LeonardD,HaskellWL, Weiner MF, Berry JD. The association between midlife cardiorespiratory fitness levels and later-life dementia: a cohort study. Ann Intern Med 2013 Feb 5;158(3):162-168.

6. Lakoski SG, Willis BL, Barlow CE, Leonard D, Gao A, Radford NB, Farrell SW, Douglas PS, Berry JD, DeFina LF, et al. Midlife cardiorespiratory fitness, incident cancer, and survival after cancer in men: the Cooper Center Longitudinal Study. JAMA Oncol 2015 May:1(2):231-237.

7. BlairSN,KohlHW3rd,PaffenbargerRSJr,ClarkDG,CooperKH, Gibbons LW. Physical fitness and all-cause mortality: a prospective study of healthy men and women. JAMA 1989 Nov 3;262(17):2395-2401.

8. Zhu W, Hooker SP, Sun Y, Xie M, Su H, Cao J. Associations of cardiorespiratory fitness with cardiovascular disease risk factors in middle-aged Chinese women: a cross-sectional study. BMC Womens Health 2014 May 1;14:62.

9. Sawada SS, Lee IM, Naito H, Kakigi R, Goto S, Kanazawa M, Okamoto T, Tsukamoto K, Muto T, Tanaka H, et al. Cardiorespiratory fitness, body mass index, and cancer mortality: a cohort study of Japanese men. BMC Public Health 2014 Sep 27;14:1012.

10. Muller J, Chan K, Myers JN. Association between exercise capacity and late onset of dementia, Alzheimer disease, and cognitive impairment. Mayo Clinic Proc 2017 Feb;92(2): 211-217.

11. GulatiM,Pandey DK, ArnsdorfMF,LauderdaleDS, Thisted RA, Wicklund RH, Al-Hani AJ, Black HR. Exercise capacity and the risk of death in women: the St James Women Take Heart Project. Circulation 2003 Sep 30;108(13):1554-1559.

12. Lyerly GW, Sui X, Lavie CJ, Church TS, Hand GA, Blair SN. The association between cardiorespiratory fitness and risk of all-cause mortality among women with impaired fasting glucose or undiagnosed diabetes mellitus. Mayo Clin Proc 2009 Sep;84(9):780-786.

13. Farrell SW, Finley CE, Radford NB, Haskell WL. Cardiorespiratory fitness, body mass index, and heart failure mortality in men: Cooper Center Longitudinal Study. Circ Heart Fail 2013 Sep 1;6(5):898-905.

14. Willis BL, Gao A, Leonard D, Defina LF, Berry JD. Midlife fitness and the development of chronic conditions in later life. Arch Intern Med 2012 Sep 24;172(17):1333-1340.

15. Schmid D, Leitzmann MF. Cardiorespiratory fitness as predictor of cancer mortality: a systematic review and metaanalysis. Ann Oncol 2015 Feb;26(2):272-278.

16. Vigen R, Colby Ayers A, Willis B, DeFina L, Berry JD. Association of cardiorespiratory fitness with total, cardiovascular, and noncardiovascular mortality across 3 decades of followup in men and women. Circ Cardiovasc Qual Outcomes 2012 May;5(3):358-364.
17. Kaminsky LA, Arena R, Beckie TM, Brubaker PH, Church TS, Forman DE, Franklin BA, Gulati M, Lavie CJ, Myers J, et al. The importance of cardiorespiratory fitness in the United States: the need for a national registry: a policy statement from the American Heart Association. Circulation $2013 \mathrm{Feb}$ 5;127(5):652-662.

18. Ross R, Blair SN, Arena R, Church TS, Després JP, Franklin BA, Haskell WL, Kaminsky LA, Levine BD, Lavie CJ, et al. Importance of assessing cardiorespiratory fitness in clinical practice: a case for fitness as a clinical vital sign: a scientific statement from the American Heart Association. Circulation 2016 Dec 13;134(24):e653-e699.

19. Guazzi M, Adams V, Conraads V, Halle M, Mezzani A, Vanhees L, Arena R, Fletcher GF, Forman DE, Kitzman DW, et al. EACPR/AHA scientific statement: clinical recommendations for cardiopulmonary exercise testing data assessment in specific patient populations. Circulation $2012 \mathrm{Oct}$ 30;126(18):2261-2274.

20. Balady GJ, Arena R, Sietsema K, Myers J, Coke L, Fletcher GF, Forman D, Franklin B, Guazzi M, Gulati M, et al. Clinician's guide to cardiopulmonary exercise testing in adults: a scientific statement from the American Heart Association. Circulation 2010 Jul 13;122(2):191-225.

21. Kaminsky LA, Arena R, Myers J. Reference standards for cardiorespiratory fitness measured with cardiopulmonary exercise testing: data from the Fitness Registry and the Importance of Exercise National Database. Mayo Clin Proc 2015 Nov;90(11):1515-1523.

22. Bouchard C, Sarzynski MA, Rice TK, Kraus WE, Church TS, Sung YJ, Rao DC, Rankinen T. Genomic predictors of the maximal $\mathrm{O}(2)$ uptake response to standardized exercise training programs. J Appl Physiol 2011 May;110(5):1160-1170.

23. Zadro JR, Shirley D, Andrade TB, Scurrah KJ, Bauman A, Ferreira PH. The beneficial effects of physical activity: is it down to your genes? A systematic review and meta-analysis of twin and family studies. Sports Med Open 2017 Dec;3(1):4.

24. Williams PT. Physical fitness and activity as separate heart disease risk factors: a meta-analysis. Med Sci Sports Exerc 2001 May;33(5):754-761.

25. DeFina LF, William L, Haskell WL, Willis BL, Carolyn E, Barlow CE, Finley CE, Levine BD, Cooper KH. Physical activity versus cardiorespiratory fitness: two (partly) distinct components of cardiovascular health? Prog Cardiovasc Dis 2015 Jan-Feb;57(4):324-329.

26. Lee DC, Sui X, Ortega FB, Kim YS, Church TS, Winett RA, Ekelund U, Katzmarzyk PT, Blair SN. Comparisons of leisure-time physical activity and cardiorespiratory fitness as predictors of all-cause mortality in men and women. Br J Sports Med 2011 May;45(6):504-510.

27. Myers J, Kaykha A, George S, Abella J, Zaheer N, Lear S, Yamazaki T, Froelicher V. Fitness versus physical activity patterns in predicting mortality in men. Am J Med 2004 Dec 15;117(12):912-918.

28. Davidson T, Vainshelboim S, Kokkinos P, Myers J, Ross, R. Cardiorespiratory fitness versus physical activity as predictors of all-cause mortality in men. Am Heart J 2017 Sep.

29. Franklin SS, Gustin W 4th, Wong ND, Larson MG, Weber MA, Kannel WB, Levy D. Hemodynamic patterns of age-related changes in blood pressure: the Framingham Heart Study. Circulation 1997 Jul 1;96(1):308-315.

30. Lewington S, Clarke R, Qizilbash N, Peto R, Collins R, Prospective Studies Collaboration. Age-specific relevance of 
usual blood pressure to vascular mortality: a meta-analysis of individual data for one million adults in 61 prospective studies. Lancet 2002 Dec 14;360(9349):1903-1913.

31. Gurven M, Blackwell AD, Rodríguez DE, Stieglitz J, Kaplan H. Does blood pressure inevitably rise with age? Longitudinal evidence among forager-Horticulturalists. Hypertension 2012 Jul;60(1):25-33.

32. Kokkinos P. Cardiorespiratory fitness, exercise, and blood pressure. Hypertension 2014 Dec;64(6):1160-1164.

33. Weintraub WS, Daniels SR, Burke LE, Franklin BA, Goff DC Jr, Hayman LL, Lloyd-Jones D, Pandey DK, Sanchez EJ, Schram AP, et al. Value of primordial and primary prevention for cardiovascular disease: a policy statement from the American Heart Association. Circulation 2011 Aug 23;124(8):967-990.

34. Kokubo Y. Prevention of hypertension and cardiovascular diseases a comparison of lifestyle factors in Westerners and East Asians. Hypertension 2014 Apr;63(4):655-660.

35. Barlow CE, Lamonte MJ, Fitzgerald SJ, Kampert JB, Perrin JL, Blair SN. Cardiorespiratory fitness is an independent predictor of hypertension incidence among initially normotensive healthy women. Am J Epidemiol 2006 Jan 15;163(2): 142-150.

36. Liu J, Sui X, Lavie CJ, Zhou H, Park YM, Cai B, Liu J, Blair SN. Effects of cardiorespiratory fitness on blood pressure trajectory with aging in a cohort of healthy men. J Am Coll Cardiol 2014 Sep 23;64(12):1245-1253.

37. Shook RP, Lee DC, Sui X, Prasad V, Hooker SP, Church TS, Blair SN. Cardiorespiratory fitness reduces the risk of incident hypertension associated with a parental history of hypertension. Hypertension 2012 Jun;59(6):1220-1224.

38. Carnethon MR, Evans NS, Church TS, Lewis CE, Schreiner PJ, Jacobs DR Jr, Sternfeld B, Sidney S. Joint associations of physical activity and aerobic fitness on the development of incident hypertension: coronary artery risk development in young adults. Hypertension 2010 Jul;56(1):49-55.

39. Juraschek SP, Blaha MJ, Whelton SP, Blumenthal R, Jones SR, Keteyian SJ, Schairer J, Brawner CA, Al-Mallah MH. Physical fitness and hypertension in a population at risk for cardiovascular disease: the Henry ford ExercIse testing (FIT) project. J Am Heart Assoc 2014 Dec;3(6):e001268.

40. Egan BM, Stevens-Fabry S. Prehypertension - prevalence, health risks, and management strategies. Nat Rev Cardiol 2015 May;12(5):289-300.

41. Sipahi I, Swaminathan A, Natesan V, Debanne SM, Simon DI, Fang JC. Effect of antihypertensive therapy on incident stroke in cohorts with prehypertensive blood pressure levels: a meta-analysis of randomized controlled trials. Stroke 2012 Feb;43(2):432-440.

42. Huang Y, Su L, Cai X, Mai M, Wang S, Hu Y, Wu Y, Tang H, $\mathrm{Xu}$ D. Association of all-cause and cardiovascular mortality with prehypertension: a meta-analysis. Am Heart J 2014 Feb;167(2):160-168.e1.

43. Faselis C, Doumas M, Kokkinos JP, Panagiotakos D, Kheirbek R, Sheriff HM, Hare K, Papademetriou V, Fletcher R, Kokkinos P. Exercise capacity and progression from prehypertension to hypertension. Hypertension 2012 Aug;60(2):333-338.

44. Sui X, Sarzynski MA, Lee D, Lavie CJ, Zhang J, Kokkinos PF, Payne J, Blair SN. Longitudinal patterns of cardiorespiratory fitness predict the development of hypertension among men and women. Am J Med 2017 Apr;130(4):469-476.e2.

45. Jae SY, Heffernan KS, Yoon ES, Park SH, Carnethon MR, Fernhall B, Choi YH, Park WH. Temporal changes in cardiorespiratory fitness and the incidence of hypertension in initially normotensive subjects. Am J Hum Biol 2012 Nov-Dec;24(6): 763-767.

46. Jae SY, Kurl S, Franklin BA, Laukkanen JA. Changes in cardiorespiratory fitness predicts incident hypertension: a population-based long-term study. Am J Hum Biol 2017 May 6;29(3):e22932.

47. Myers J,Prakash M, Froelicher V, DoD, PartingtonS, AtwoodJE. Exercise capacity and mortality among men referred for exercise testing. N Engl J Med 2002 Mar 14;346(11):793-801.

48. Faselis C, Doumas M, Panagiotakos D, Kheirbek R, Korshak L, Manolis A, Pittaras A, Tsioufis C, Papademetriou V, Fletcher RD, et al. Body mass index, exercise capacity, and mortality risk in male veterans with hypertension. Am J Hypertens 2012 Apr;25(4):444-450.

49. Evenson KR, Stevens J, Thomas R, Cai J. Effect of cardiorespiratory fitness on mortality among hypertensive and normotensive women and men. Epidemiology 2004 Sep;15(5):565-572.

50. Sui X, Sarzynski MA, Lee D, Kokkinos PF. Impact of changes in cardiorespiratory fitness on hypertension, dyslipidemia and survival: an overview of the epidemiological evidence. Prog Cardiovasc Dis 2017 Jun-Jul;60(1):56-66.

51. Mora S, Cook N, Buring JE, Ridker PM, Lee IM. Physical activity and reduced risk of cardiovascular events: potential mediating mechanisms. Circulation 2007 Nov 6;116(19): 2110-2118.

52. Green DJ, O'Driscoll G, Joyner MJ, Cable NT. Exercise and cardiovascular risk reduction: time to update the rationale for exercise? J Appl Physiol 2008 Aug;105(2):766-768.

53. Franklin BA. Mayo Cardiorespiratory fitness: an independent and additive marker of risk stratification and health outcomes. Mayo Clin Proc 2009 Sep;84(9):776-779.

54. Carrick-Ranson G, Hastings JL, Bhella PS, Fujimoto N, Shibata S, Palmer MD, Boyd K, Livingston S, Dijk E, Levine BD. The effect of lifelong exercise dose on cardiovascular function during exercise. J Appl Physiol 2014 Apr 1;116(7):736-745.

55. Nauman J, Nes B, Lavie C, Jackson AS, Sui X, Coombes JS, Blair SN, Wisløff U. Prediction of cardiovascular mortality by estimated cardiorespiratory fitness independent of traditional risk factors: the HUNT study. Mayo Clin Proc 2017 Feb;92(2):218-227.

56. Physical Activity Guidelines Advisory Committee. Physical Activity Guidelines Advisory Committee Report, 2008. Washington (DC): U.S. Department of Health and Human Services; 2008. Accessed 2017 Sep 21. Available from: http:// www.health.gov/paguidelines. 